\title{
GENERALIZED LIE DERIVATIONS OF UNITAL ALGEBRAS WITH IDEMPOTENTS
}

\section{DOMINIK BENKOVIČ}

Abstract. Let $\mathscr{A}$ be a unital algebra with a nontrivial idempotent $e$ over a unital commutative ring $R$. We show that under suitable assumptions every generalized Lie $n$-derivation $F: \mathscr{A} \rightarrow$ $\mathscr{A}$ is of the form $F(x)=\lambda x+\Delta(x)$, where $\lambda \in Z(\mathscr{A})$ and $\Delta$ is a Lie $n$-derivation of $\mathscr{A}$. As an application, we give a description of generalized Lie $n$-derivations on classical examples of unital algebras with idempotents: triangular algebras, matrix algebras, nest algebras and algebras of all bounded linear operators.

Mathematics subject classification (2010): 16W25, 15A78, 47L35.

Keywords and phrases: Generalized Lie $n$-derivation, Lie $n$-derivation, derivation, unital algebra, triangular algebra.

\section{REFERENCES}

[1] M. Ashraf, A. JABeEn, Nonlinear generalized Lie triple derivation on triangular algebras, Communications in Algebra 45 (2017), 4380-4395.

[2] D. BenKovič, Generalized Lie derivations on triangular algebras, Linear Algebra Appl. 434 (2011), $1532-1544$.

[3] D. BEnKovič, Lie triple derivations of unital algebras with idempotents, Linear Multilinear Algebra 63 (2015), 141-165.

[4] D. Benkovič, D. ERemita, Multiplicative Lie n-derivations of triangular rings, Linear Algebra Appl. 436 (2012), 4223-4240.

[5] W.-S. Cheung, Lie derivations of triangular algebras, Linear Multilinear Algebra 51 (2003), 299 310.

[6] Y. Du, Y. WANG, Lie derivations of generalized matrix algebras, Linear Algebra Appl. 437 (2012), 2719-2726.

[7] X. H. FeI, J. H. ZHANG, Nonlinear generalized Lie derivations on triangular algebras, Linear Multilinear Algebra 65 (2017), 1158-1170.

[8] P. Ji, R. LiU, Y. ZHAO, Nonlinear Lie triple derivations of triangular algebras, Linear and Multilinear Algebra 60 (2012), 1155-1164.

[9] P. S. JI, W. Q. QI, Charactrizations of Lie derivations of triangular algebras, Linear Algebra Appl. 435 (2011) 1137-1146.

[10] F. LU, Lie triple derivations on nest algebras, Math. Nachr. 280 (2007), 882-887.

[11] Z.-K XIAO, F. WeI, Lie triple derivations of triangular algebras, Linear Algebra Appl. 437 (2012), $1234-1249$.

[12] W.-Y. YU, J.-H. ZhANG, Nonlinear Lie derivations of triangular algebras, Linear Algebra Appl. 432 (2010), 2953-2960.

[13] Y. WANG, Lie n-derivations of unital algebras with idempotents, Linear Algebra Appl. 458 (2014), $512-525$.

[14] Y. WANG, Y. WANG, Multiplicative Lie $\mathrm{n}$-derivations of generalized matrix algebras, Linear Algebra Appl. 438 (2013), 2599-2616.

[15] J. H. ZhANG, B. W Wu, H. X. CAO, Lie triple derivations of nest algebras, Linear Algebra Appl. 416 (2006), 559-567. 